\title{
CORRIGENDUM
}

\section{Motivation and challenge to capture both large scale and local transport in next generation accretion theory - CORRIGENDUM}

\author{
Eric G. Blackman and Farrukh Nauman \\ doi:10.1017/S0022377815000999, Published by Cambridge University Press, \\ October 1, 2015
}

In the first paragraph of $\S 4.4 .1$ of our recently published manuscript (Blackman \& Nauman 2015) there is a typographical error that may cause confusion: the $b_{x}$ should be interchanged with $b_{y}$ to match the rest of the text. The corrected paragraph is below:

\subsubsection{Tilt angle from fluctuating fields}

Because the magnetic stress dominates the Reynolds stress we have, for the box averaged contribution from fluctuations

$$
\alpha_{s s} \simeq \frac{\left\langle b^{2}\right\rangle}{P} \frac{\left\langle b_{x} b_{y}\right\rangle}{\left\langle b^{2}\right\rangle}=\beta^{-1} \frac{\tan \theta}{\left(1+\tan ^{2} \theta+b_{z}^{2} / b_{y}^{2}\right)} \sim \beta^{-1} \tan \theta,
$$

where $\tan \theta=b_{x} / b_{y}$, and we assume $\left|b_{z} / b_{y}\right| \ll 1$ and $\tan \theta \ll 1$ for the last similarity (justified later).

\section{REFERENCES}

Blackman, E. G. \& Nauman, F. 2015 Motivation and challenge to capture both large-scale and local transport in next generation accretion theory. J. Plasma Phys. 81 (5), 395810505. 